\title{
A Case Report of Negative Pressure Wound Therapy in Post- Traumatic Raw Area
}

\author{
Naim Sulaiman Abuzarifa* (D) and Wan Azman Wan Sulaiman \\ Reconstructive Sciences Unit, PPSP Health Campus, Universiti Sains Malaysia (USM), Malaysia

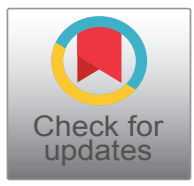

*Corresponding author: Dr. Naim Sulaiman Abuzarifa, Reconstructive Sciences Unit, PPSP Health Campus, Universiti Sains Malaysia (USM), Kubang Kerian, 16150, Kelantan, Malaysia, Tel: 00601127586785

\begin{abstract}
Traumatic wounds vary from abrasions and minor skin incisions or tears to wounds with extensive tissue damage or loss and damage to bone and internal organs. The use of economic negative-pressure wound therapy for managing wounds and negative-pressure wound therapy is known for improving a wide range of acute and chronic wounds. This medical device involves applying a wound dressing through which negative pressure is applied and wound discharges drawn out from the wound. The treatment aims to prepare wounds for further surgery, reduce the rate of infection, and potentially decrease the time of wound healing. This technique has historical origins that go back to ancient times. Many concepts were hypothesized that wound care method includes rapid draining of the wound discharge, reducing the bacterial load of the wound, mechanical traction of wound edges by negative pressure and induction of wound surface hypoxia that activate local angiogenesis. Using $\mathrm{Ne}$ gative-pressure wound therapy to show our method's efficiency for wound care and healing will be presented. This case aims to present a 17-year-old Malay female patient present with post road traffic accident, presented to our department 18 days after the incident with a left leg injury with the sustained open comminuted left distal tibia and fibula fracture repaired by ortho team and skin and soft tissue loss at the same spot with the application of therapy is a relatively simple technique that should be taken into account when considering for treating open traumatic wounds to decreased bacterial burden as well as to prepare the wound for Skin graft and to reduce the hospitalization time.
\end{abstract}

\section{Keywords}

Wounds, Negative pressure wound therapy, Trauma

\section{Introduction}

The Negative-pressure wound therapy (NPWT) appears in the 1990s. However, using suction to treat the wounds goes back to old history in the Roman era. During the Soviet Afghan war (1979-1989), Russians developed suction devices and foam to treat infected wounds. NPWT therapy was presented by Argenta and Morykwas of Wake Forest University School of Medicine in the nineties. Different theories and mechanisms were proposed to clarify the healing potentials of negative pressure wound therapy technique. Negative pressure applies forces on the wound when applied in a sealed method, leading to a mechanical reduction in the wound size as the mechanical force generated by the negative pressure is transmitted to the cells through the extracellular matrix, leading to microdeformation of the cytoskeleton that stimulates cell proliferation through mechanotransduction. These cellular changes lead to undulant wound surface due to negative suction at the foam-tissue interface. Angiogenesis stimulation Surface hypoxia induced by negative pressure and vascular endothelial growth factor (VEGF) expression and mechanical forces were linked with the formation of more physiological and straight blood vessels at the wound site. NPWT removes excess wound fluids and reduces oedema. This mechanism explains low levels of anti-inflammatory mediators, e.g. metalloproteinase, which breaks down adhesion proteins responsible for wound healing. Wounds treated with NPWT showed a faster reduction in bacterial colonization, which can be explained by either reduced wound discharge that decreased bacterial colonization or prevents the wound

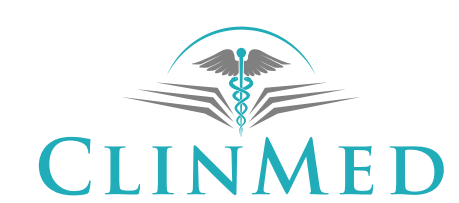

INTERNATIONAL LIBRARY 
against outer environmental contamination, variables in applications Pressure, Morykwas proved that -125 $\mathrm{mmHg}$ increased blood flow in swine wound models. As well as flow Mode as Continuous mode was found to cause less pain. However, the intermittent mode was found to induce granulation faster but cause more pain and leakage of the discharge into the wound during an intermittent period. NPWT dressing change Varies from two to four days as time gaps between dressings [1,2]. NPWT is indicated for wound closure and to prepare the wound bed before surgery, as an aid for successful graft take and adjunct therapy in wound reconstruction using a dermal substitute. On the other hand, it is contraindicated in wounds with very poor vascularity, deeper tissues infections, e.g. osteomyelitis, malignancy, fistulas and wounds with necrotic tissues and eschars [3].

\section{Case}

The case can be briefly presented as a 17-year-old female patient, post road traffic accident, presented to our department 18 days after the incident with a left leg injury with the sustained open comminuted left distal tibia and fibula fracture repaired by orthopaedic team and skin and soft tissue loss at the same spot.

Patient with history of malaise and disturbed appetite and On examination post-traumatic vertical defect at the posterior aspect of the distal part of the left leg about $20 \times 15 \mathrm{~cm}$ exposed some part of Achilles Tendon and distal part of the gastrocnemius muscle, with pus discharge, a Blood test with high ESR and CRP (Figure 1).

Wound swab for culture and sensitivity showed growth of pseudomonas, NPWT was applied by assembled device, and it is made up of major components which are, a connecting tube, a vacuum system, a semipermeable dressing to isolate the wound environment and allow the vacuum system to transmit sub-atmospheric pressure to the wound surface and a sponge of the polyurethane black hydrophobic foam made of many cells that are highly interconnected with an average pore size of 400 to $600 \mu \mathrm{m}$, it is placed into the wound as well as this type of foam generally used for wounds with extensive fluid drainage and to stimulate granulation tissue formation. This permits the vacuum to be distributed evenly throughout the foam and improves drainage. Typically, the NPWT system is changed every two to three days, making the wound less susceptible to cross-contamination than the average level of contamination when the dressing is changed 2 or 3 times a week, Pressure applied was $-125 \mathrm{mmHg}$, according to Morykwas recommendations, and the mode is continuous. Healthy and clean granulation tissue was formed, the wound was ready for skin graft after three cycles of NPWT, and Wound swab for culture and sensitivity showed no growth (Figure 2 and Figure 3).

\section{Discussion}

The effect of NPWT on bacterial load remains controversial. Some studies have shown a reduced bacterial load in response to NPWT. Others study comparable levels between treatment and control groups, comparing foam dressings with and without suction, respectively [4]. However, this result was in the context of an in vitro analysis using non-viable tissue which suggests that any

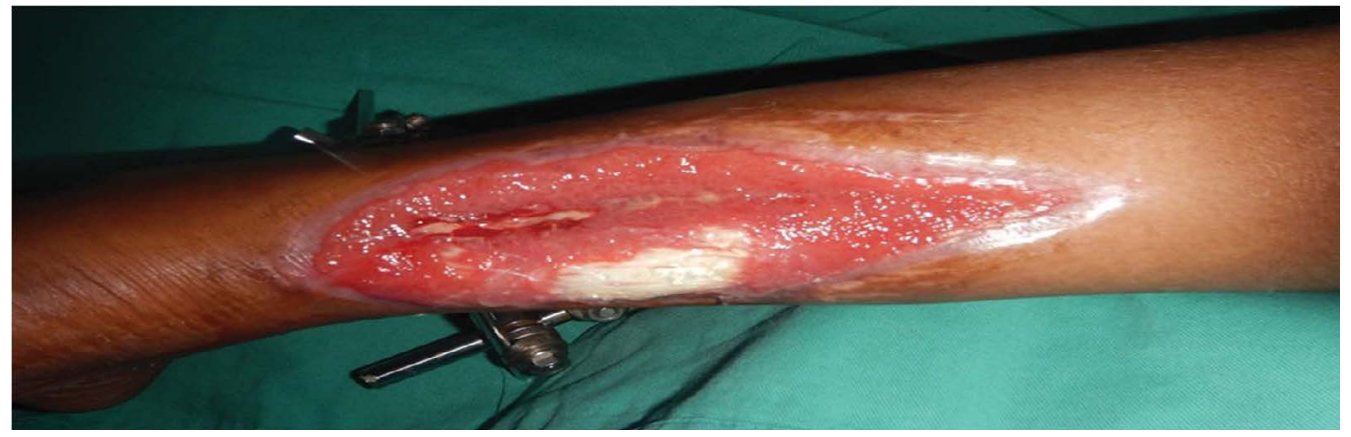

Figure 1: The wound in the posterior aspect of left leg after first cycle of NPWT

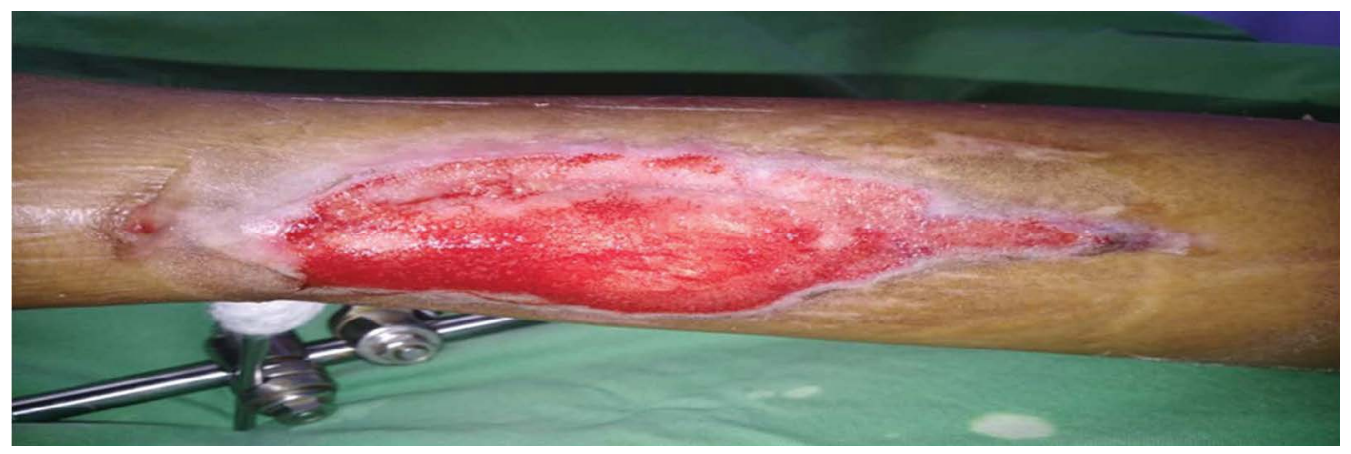

Figure 2: The wound after second cycle of NPWT 


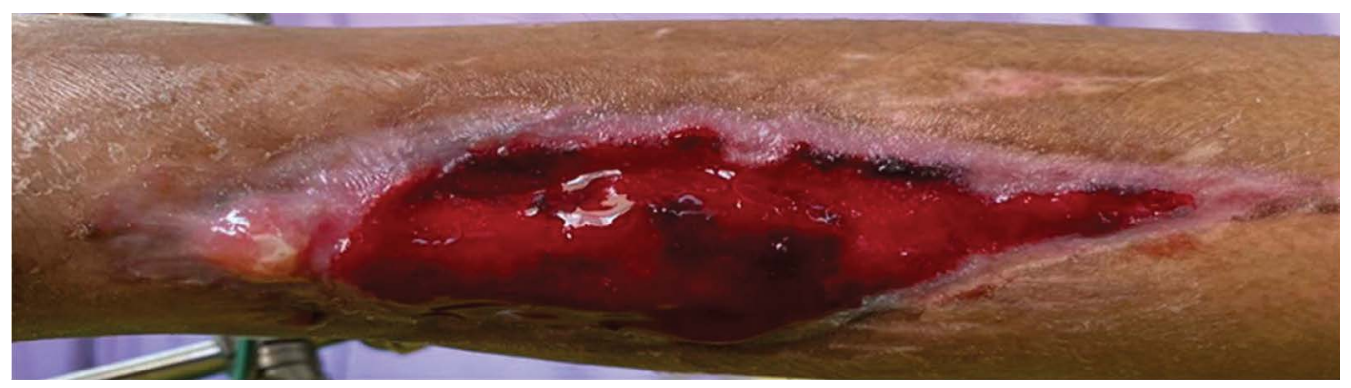

Figure 3: The wound after third cycle of NPWT

observation of reduced bacterial load results from more than physical suction. Another study observed a decrease in non-fermentative gram-negative bacilli, while the quantity of Staphylococcus aureus increased. The effect of NPWT on bacterial load remains an area to discover further. High bacterial levels have been measured in sonicated foams. Porous polyurethane ether foam on high suction pressure had fewer bacteria than polyvinyl alcohol foams on lower suction pressure [5]. Earlier application of NPWT to these wounds also results in faster healing times. In the treatment of diabetic foot ulcers, NPWT decrease wound area, promote wound bed granulation, and microbial clearance, thereby enabling a higher rate of limb salvage, especially in Wagner grade 3 and grade 4 ulcers. NPWT may protect the amputation of digits in the treatment of scleroderma ulcers [6]. Applications NPWT have been applied to different wounds varying in location, difficulty, and underlying disease. It alters the traditional phases of wound healing and is used in many anatomical sites in the body. The original application of suction to wounds seems to be a straightforward concept. Open wounds applications of NPWT Classically, NPWT has been used in an open wound. In treating pressure ulcers, serial randomized controlled trials show a reduction in wound surface area, depth, improved granulation, and reduced hospitalization frequency in patients undergoing NPWT. This therapy can be an option for managing nonhealing deep-pressure ulcers covered by soft necrotic tissue; wounds treated with NPWT often show a rapid granulation tissue formation. In a retrospective cohort study evaluating chronic diabetic, arterial, and venous ulcers in high-risk patients, treatment with NPWT increased closure incidence [5]. NPWT could be applied in different posttraumatic wounds to reduced bacterial load, as shown in our case.

\section{Conclusion}

NPWT is the principle of treatment gives significantly positive results of improvement in the wound, as shown in the case report. Application of the NPWT was a useful adjunct in treating and stimulating wound healing in our case. This is a case report of its kind utilizing wound VAC with NPWT, with successful results showing a decreased bacterial burden and patient wound closure.

\section{References}

1. Iheozor-Ejiofor Z, Newton K, Dumville JC, Costa ML, Norman G, et al. (2018) Negative pressure wound therapy for open traumatic wounds. Cochrane Database Syst Rev 7: CD012522.

2. Abdelmoez A (2018) The use of economic negative-pressure wound therapy (NPWT) therapy machine and supplies in management of acute and chronic wounds: A report of 3 cases. Surg Case Rep 2: 39-41.

3. Novak A, Khan WS, Palmer J (2014) The evidence-based principles of negative pressure wound therapy in trauma \& orthopedics. Open Orthop J 8: 168-177.

4. Patmo AS, Krijnen P, Tuinebreijer WE, Breederveld RS (2014) The effect of vacuum-assisted closure on the bacterial load and type of bacteria: A systematic review. Adv Wound Care 3: 383-389.

5. Huang C, Leavitt T, Bayer LR, Orgill DP (2014) Effect of negative pressure wound therapy on wound healing. Curr Probl Surg 51: 301-331.

6. Hasan MY, Teo R, Nather A (2015) Negative-pressure wound therapy for management of diabetic foot wounds: A review of the mechanism of action, clinical applications, and recent developments. Diabetic Foot Ankle 6: 27618. 